\title{
$\mathrm{CH}_{3} \mathrm{COONa}$ 处理 HZSM-5 分子篮及其催化性能
}

\author{
徐国皓 ${ }^{1}$, 余金鹏 ${ }^{1,2}$, 徐华胜 ${ }^{1,2}$, 李春成 ${ }^{1,2}$, 黄金花 ${ }^{1}$, 王鹏飞 ${ }^{1,2}$ \\ (1. 上海化工研究院有限公司，上海 200062; 2. 上海绿强新材料有限公司，上海 201608)
}

摘 要: 采用不同浓度的 $\mathrm{CH}_{3} \mathrm{COONa}$ 溶液和常规碱 $\left(\mathrm{NaOH}\right.$ 和 $\left.\mathrm{Na}_{2} \mathrm{CO}_{3}\right)$ 溶液处理 $\mathrm{HZSM}-5$ 分子篮, 对处理前后的 HZSM-5 分子篮负载 Pt, 制备 Pt/HZSM-5 催化剂用于丙烷脱氢反应。利用 X 射线衍射 $(X R D) 、 X$ 射线荧光光谱 $(X R F) 、$ 扫描电镜(SEM)、比表面测试仪(BET)、 $\mathrm{NH}_{3}-\mathrm{TPD}$ (程序升温脱附法)和 $\mathrm{H}_{2}$ 化学吸附等手段对处理前后的 HZSM-5 分 子篮进行表征, 并考察了不同浓度的 $\mathrm{CH}_{3} \mathrm{COONa}$ 溶液和常规碱溶液处理对 HZSM-5 分子篮的晶体结构、表面形貌、 孔结构、表面酸性及丙烷脱氢性能的影响。结果表明, $\mathrm{CH}_{3} \mathrm{COONa}$ 溶液具有碱改性作用, 与常规碱溶液相比, 采用 $\mathrm{CH}_{3} \mathrm{COONa}$ 溶液处理 HZSM-5 分子篎, 能够在对分子篎的晶体结构和表面形貌影响较小的基础上有效地引入介孔结 构, 具有较好的可调控性; 随着 $\mathrm{CH}_{3} \mathrm{COONa}$ 溶液浓度的增加, 丙烷脱氢反应的丙烷初始转化率和丙烯选择性先升高 后降低, 当 $\mathrm{CH}_{3} \mathrm{COONa}$ 溶液浓度为 $4.0 \mathrm{~mol} / \mathrm{L}$ 时, 丙烷初始转化率和丙烯选择性均达到最高值, 分别为 $34.5 \%$ 和 $98.9 \%$ 。

关 键 词: HZSM-5; 碱处理; 丙烷脱氢

中图分类号: TQ630 文献标识码: A

\section{Catalystic Performance of HZSM-5 Zeolite Treated by $\mathrm{CH}_{3} \mathrm{COONa}$}

\author{
XU Guo-Hao ${ }^{1}$, YU Jin-Peng ${ }^{1,2}$, XU Hua-Sheng ${ }^{1,2}$, LI Chun-Cheng ${ }^{1,2}$, HUANG Jin-Hua ${ }^{1}$, WANG Peng-Fei ${ }^{1,2}$ \\ (1. Shanghai Research Institute of Chemical Industry Co., Ltd, Shanghai 200062, China; 2. Shanghai Lüqiang New Materials \\ Co., Ltd, Shanghai 201608, China)
}

\begin{abstract}
HZSM-5 zeolites were treated by $\mathrm{CH}_{3} \mathrm{COONa}$ solution with different concentrations and conventional alkali $\left(\mathrm{NaOH}\right.$ or $\left.\mathrm{Na}_{2} \mathrm{CO}_{3}\right)$ solution. Then, Pt was loaded on HZSM-5 zeolites before and after the treatment to synthesize Pt/HZSM-5 catalysts for propane dehydrogenation reaction. HZSM-5 zeolites were characterized by XRD, XRF, SEM, BET, $\mathrm{NH}_{3}$-TPD, and $\mathrm{H}_{2}$ chemisorption. The effect of $\mathrm{CH}_{3} \mathrm{COONa}$ solution with different concentrations and conventional alkali solution on crystal structure, surface morphology, pore structure, surface acidity, and propane dehydrogenation performance of HZSM-5 zeolites were investigated. The results showed that $\mathrm{CH}_{3} \mathrm{COONa}$ solution modified HZSM- 5 zeolites by alkali. Compared with conventional alkali solution, the treatment of $\mathrm{CH}_{3} \mathrm{COONa}$ solution for HZSM-5 zeolites could more effectively and better controllably introduce the mesoporous structure with less influence on the crystal structure and surface morphology. With increase of the concentration of $\mathrm{CH}_{3} \mathrm{COONa}$ solution, the initial propane conversion and the propene selectivity in propane dehydrogenation firstly increased and then decreased. The catalyst treated by $4.0 \mathrm{~mol} / \mathrm{L} \mathrm{CH}_{3} \mathrm{COONa}$ solution exhibited the highest propane initial conversion and propene selectivity, which were $34.5 \%$ and $98.9 \%$, respectively.
\end{abstract}

Key words: HZSM-5; alkali treatment; propane dehydrogenation 
丙烯是仅次于乙烯的重要化工基础原料，其衍生 物主要包括聚丙烯、异丙醇、丙酮、丙烯腈等 ${ }^{[1]}$, 而随 着具有耐热、质轻、强度好等特点的聚丙烯广泛用于 包装、家电、汽车和建筑等领域, 使得聚丙烯的需求 量增长迅速, 作为原料的丙烯需求量也随之快速增 长。目前工业上主要有四种途径增产丙烯: 一是催化 裂化(FCC)装置升级, 二是烯烃歧化, 三是 C4/C5 烃选 择性裂解, 四是丙烷脱氢工艺。根据我国能源结构特 点, 开发高产且廉价的丙烷催化脱氢制丙烯技术, 对 于缓解丙烯供需矛盾、优化能源和化工产业结构等具 有重要战略意义。

现已实现工业化的丙烷脱氢制丙烯工艺中, 主要 以 $\mathrm{Al}_{2} \mathrm{O}_{3}$ 作为催化剂载体, 以 $\mathrm{Pt}$ 作为活性组分, 其丙 烷单程转化率为 $30 \%$, 丙烯选择性为 $84 \%{ }^{[2]}$, 虽然该工 艺具有较高的丙烷脱氢反应活性, 但由于 $\mathrm{Al}_{2} \mathrm{O}_{3}$ 载体孔 径较大, 丙烯选择性差, 催化性能难以令人满意 ${ }^{[3]}$ 。 ZSM-5 分子篮含有双十圆环交叉的孔道体系, 孔径较 小, 具有优异的择形催化性能 ${ }^{[4-5]}$, 因此, ZSM-5 分子 篮是替代 $\mathrm{Al}_{2} \mathrm{O}_{3}$ 的优良载体。从文献[6-7]可以看出, 科 研人员大都将研究重点放在通过在 Pt/ZSM-5 分子篎 催化剂上负载助剂对催化剂进行改性, 提高丙烷脱氢 反应性能, 但对 ZSM-5 分子篮载体本身的结构调变研 究较少。

ZSM-5 分子笁作为固体酸催化剂的代表, 表面酸性 较强, 在高温环境中容易积碳导致催化剂失活, 而通过 对 ZSM-5 分子篮进行碱处理 ${ }^{[8-9]}$, 可以有效地调节分子 笁表面酸性，弥补这一缺陷，同时能够形成微孔一介孔多 级孔结构, 提高 ZSM-5 分子篮的传质能力。目前, 关 于碱处理 ZSM-5 分子笁的报道大都采用碱性较强的 $\mathrm{NaOH}$ 溶液和 $\mathrm{Na}_{2} \mathrm{CO}_{3}$ 溶液 ${ }^{[10-11]}$, 但对 $\mathrm{CH}_{3} \mathrm{COONa}$ 溶 液对 ZSM-5 分子篮进行碱处理还未见报道。

因此，本工作采用不同浓度的 $\mathrm{CH}_{3} \mathrm{COONa}$ 溶液对 HZSM-5 分子篮进行处理, 并与相关文献报道的最佳 浓度的 $\mathrm{NaOH}$ 和 $\mathrm{Na}_{2} \mathrm{CO}_{3}$ 溶液处理的分子篮 ${ }^{[12-14]}$ 进行 对比, 再在上述碱处理前后的 HZSM-5 分子篮上负载 Pt, 制备 Pt/HZSM-5 分子篮催化剂, 考察不同浓度的 $\mathrm{CH}_{3} \mathrm{COONa}$ 溶液和常规碱溶液 $\left(\mathrm{Na}_{2} \mathrm{CO}_{3}\right.$ 和 $\mathrm{NaOH}$ 溶液 $)$ 对 HZSM-5 分子篎的孔结构、形貌、表面酸性和丙烷 脱氢反应性能的影响。

\section{1 实验方法}

\section{1 试剂}

$\mathrm{NaOH} 、 \mathrm{CH}_{3} \mathrm{COONa} 、 \mathrm{Na}_{2} \mathrm{CO}_{3}$ ：分析纯，江苏强盛 功能化学股份有限公司; $\mathrm{Al}_{2}\left(\mathrm{SO}_{4}\right)_{3} \cdot 18 \mathrm{H}_{2} \mathrm{O}$ : 分析纯, 上 海展云化工有限公司; $\mathrm{TPAOH}$ : 质量分数 $25 \%$, 国药集 团化学试剂有限公司; $\mathrm{H}_{2} \mathrm{PtCl}_{6} \cdot 6 \mathrm{H}_{2} \mathrm{O}$ : 分析纯, 上海精
细材料研究所; 硅溶胶: 质量分数 $25 \%$, 上海江桥焊条 辅料有限公司。

\section{2 催化剂的制备}

以硫酸铝为铝源, 硅溶胶为硅源, 采用水热合成 法合成硅铝摩尔比为 100 的 NaZSM-5 分子篎。将得到 的 NaZSM-5 分子笁原粉与 $1 \mathrm{~mol} / \mathrm{L} \mathrm{NH}_{4} \mathrm{NO}_{3}$ 溶液按照 固液质量比 $1: 20$ 混合, 在 $80{ }^{\circ} \mathrm{C}$ 水浴搅拌 $2 \mathrm{~h}$, 洗涤 $\rightarrow$ $120{ }^{\circ} \mathrm{C}$ 烘干 $\rightarrow 550{ }^{\circ} \mathrm{C}$ 焙烧, 反复进行三次, 将 NaZSM-5 分子篮转换为 HZSM-5 分子篮。将制备的 HZSM-5 分 子篮分别与不同浓度的 $\mathrm{CH}_{3} \mathrm{COONa}$ 溶液(2.0、4.0 和 $6.0 \mathrm{~mol} / \mathrm{L}) 、 1.0 \mathrm{~mol} / \mathrm{L} \mathrm{NaOH}$ 溶液和 $2.0 \mathrm{~mol} / \mathrm{L} \mathrm{Na}_{2} \mathrm{CO}_{3}$ 溶液按照固液质量比 $1: 20$ 混合, $80{ }^{\circ} \mathrm{C}$ 水浴搅拌 $2 \mathrm{~h}$, 冰水急冷、抽滤、去离子水洗涤至中性, 经 $120{ }^{\circ} \mathrm{C}$ 干 燥 $12 \mathrm{~h}$ 。根据碱溶液种类和浓度的不同，将所得 HZSM-5 分子篮分别记为 HZSM-5 $\left(\mathrm{CH}_{3} \mathrm{COONa}, n\right)$ 、 HZSM-5(NaOH, 1.0)和 HZSM-5 $\left(\mathrm{Na}_{2} \mathrm{CO}_{3}, 2.0\right)$, 其中, $n$ 代表不同浓度的 $\mathrm{CH}_{3} \mathrm{COONa}$ 溶液; 未经碱溶液处理的 HZSM-5 分子笁记为 HZSM-5 (0)。

将处理前后样品等体积浸渍质量分数为 $0.5 \%$ 的 氯铂酸溶液中, $120{ }^{\circ} \mathrm{C}$ 烘干, $550{ }^{\circ} \mathrm{C}$ 焙烧 $4 \mathrm{~h}$, 分别得到 Pt/HZSM-5(0)、Pt/HZSM-5 $\left(\mathrm{CH}_{3} \mathrm{COONa}, n\right) 、 \mathrm{Pt} / \mathrm{HZSM}-5$ $(\mathrm{NaOH}, 1.0)$ 和 $\mathrm{Pt} / \mathrm{HZSM}-5\left(\mathrm{Na}_{2} \mathrm{CO}_{3}, 2.0\right)$ 催化剂, 其中, $n$ 代表不同浓度的 $\mathrm{CH}_{3} \mathrm{COONa}$ 溶液。

\section{3 催化剂的表征}

在 $\mathrm{D} / \mathrm{max}-2550 \mathrm{VB} / \mathrm{PC}$ 型 $\mathrm{X}$ 射线衍射 $(\mathrm{XRD})$ 仪上进 行样品的物相分析, 测试条件为: 衍射源 $\mathrm{Cu}-\mathrm{K} \alpha(\lambda=$ $0.15406 \mathrm{~nm})$, 管电压 $40 \mathrm{kV}$, 管电流 $40 \mathrm{~mA}$, 扫描范围 $5^{\circ} \sim 50^{\circ}$, 扫描速率 $2\left(^{\circ}\right) / \mathrm{min}$ 。在岛津 XRF-1700 型 $\mathrm{X}$ 射 线苂光光谱分析仪上分析样品的 $\mathrm{SiO}_{2} / \mathrm{Al}_{2} \mathrm{O}_{3}$ 物质量 比。在 Merlin Compact 型扫描电子显微镜(SEM)上观 察样品形貌。在美国麦克仪器公司制造的 ASAP2020 型物理吸附仪上进行 BET 表征, 并将高纯氮作为吸附质, 测定样品的比表面积、孔容和孔径。在 CHMBET-3000 型化学吸附仪上表征样品的表面酸性。在 TP5000-II 多 用吸附仪上表征样品的 $\mathrm{H}_{2}$ 化学吸附 ${ }^{[15]}$ 。

\section{4 催化剂的活性评价}

在自行组装的固定床微型反应装置上进行丙烷脱 氢催化剂的活性评价。丙烷脱氢评价条件：压力为 $0.1 \mathrm{MPa}$, 温度为 $590{ }^{\circ} \mathrm{C}$, 丙烷的质量空速为 $3 \mathrm{~h}^{-1}$ 。用 Agilent 公司 GC-2060 型气相色谱仪在线分析反应产物 的组成。

\section{2 结果与讨论}

\section{1 碱处理对 HZSM-5 分子篮晶体结构的影响}

图 1 为 HZSM-5 分子篮经不同碱溶液处理前后的 $\mathrm{XRD}$ 图谱, 表 1 为上述样品的相对结晶度和硅铝比。 


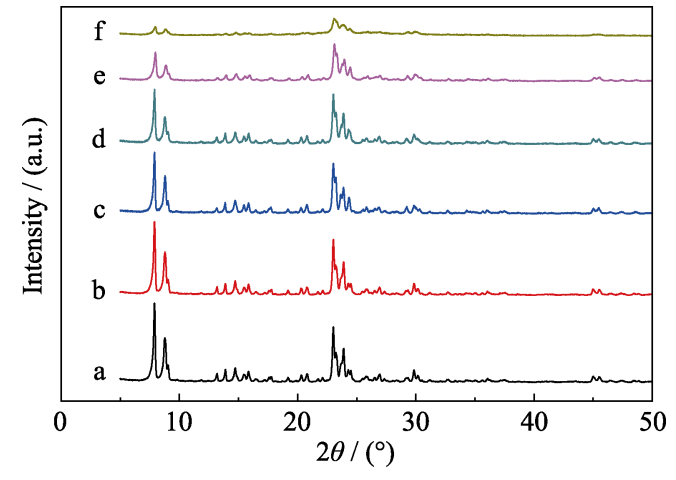

图 1 碱处理前后 HZSM-5 分子笁的 XRD 图谱

Fig. 1 XRD patterns of HZSM-5 zeolites before and after alkaline treatment

a: HZSM-5(0); b: HZSM-5( $\left.\mathrm{CH}_{3} \mathrm{COONa}, 2.0\right)$; c: HZSM-5( $\mathrm{CH}_{3} \mathrm{COONa}$, 4.0); d: HZSM-5( $\left.\mathrm{CH}_{3} \mathrm{COONa}, 6.0\right)$; e: HZSM-5 $\left(\mathrm{Na}_{2} \mathrm{CO}_{3}, 2.0\right)$; f: HZSM$5(\mathrm{NaOH}, 1.0)$

表 1 碱处理前后 HZSM-5 分子篮的相对结晶度和硅铝比

Table 1 Relative crystallinity and $n\left(\mathrm{SiO}_{2}\right) / n\left(\mathrm{Al}_{2} \mathrm{O}_{3}\right)$ of HZSM-5 zeolites before and after alkali treatment

\begin{tabular}{ccc}
\hline Sample & $\begin{array}{c}\text { Relative } \\
\text { crystallinity } / \%\end{array}$ & $\begin{array}{c}n\left(\mathrm{SiO}_{2}\right) \\
\ln \left(\mathrm{Al}_{2} \mathrm{O}_{3}\right)\end{array}$ \\
\hline HZSM-5(0) & 100 & 100 \\
HZSM-5(CH $\left(\mathrm{CH}_{3} \mathrm{COONa}, 2.0\right)$ & 99 & 94 \\
HZSM-5 $\left(\mathrm{CH}_{3} \mathrm{COONa}, 4.0\right)$ & 97 & 90 \\
HZSM-5 $\left(\mathrm{CH}_{3} \mathrm{COONa}, 6.0\right)$ & 85 & 82 \\
HZSM-5 $\left(\mathrm{Na}_{2} \mathrm{CO}_{3}, 2.0\right)$ & 62 & 74 \\
HZSM-5 $(\mathrm{NaOH}, 1.0)$ & 24 & 63 \\
\hline
\end{tabular}

由图 1 可知, 不同碱溶液处理前后, 各样品在 $2 \theta=7.9^{\circ}$ 、 $8.8^{\circ} 、 23.7^{\circ}$ 和 $24.4^{\circ}$ 处均有特征衍射峰出现, 说明经过 不同碱溶液处理后的样品，仍保留了 HZSM-5 分子篮 的晶相结构。由表 1 可见, $\mathrm{NaOH}$ 溶液对分子䇻的结晶 度和硅铝比影响最大，当采用 $1.0 \mathrm{~mol} / \mathrm{L} \mathrm{NaOH}$ 溶液处 理 HZSM-5 分子篮时, HZSM-5 分子篮的相对结晶度仅
为 $24 \%$, 硅铝比仅为 $63 \% ; \mathrm{Na}_{2} \mathrm{CO}_{3}$ 溶液对 HZSM-5 分 子篎的结晶度和硅铝比影响次之; $\mathrm{CH}_{3} \mathrm{COONa}$ 溶液对 HZSM-5 分子篮的结晶度和硅铝比影响最小, 且随着 $\mathrm{CH}_{3} \mathrm{COONa}$ 溶液浓度的增加，相对结晶度和硅铝比逐 渐减小。这是因为碱处理 HZSM-5 分子笁主要脱除分 子篮内的骨架硅，对骨架上铝的脱除作用较小，且随 着碱性的增强, 脱硅深度不断加深, 又由于 $\mathrm{NaOH}$ 溶 液和 $\mathrm{Na}_{2} \mathrm{CO}_{3}$ 溶液的碱性较强, 对分子篎的骨架结构 腐蚀比较严重, 所以 HZSM-5 分子篎的结晶度和硅铝 比变化较大。而 $\mathrm{CH}_{3} \mathrm{COONa}$ 溶液碱性较弱, 对分子篮 骨架结构腐蚀程度很小, 因此能够保持较高的相对结 晶度和硅铝比 ${ }^{[16]}$, 且随着 $\mathrm{CH}_{3} \mathrm{COONa}$ 溶液浓度的增加, 脱硅深度不断加深, 因此, 随着 $\mathrm{CH}_{3} \mathrm{COONa}$ 溶液浓度 的增加, 处理后的 HZSM-5 分子耖的相对结晶度和硅 铝比逐渐降低。

\section{2 碱处理对 HZSM-5 分子篮表面形貌的 影响}

图 2 为碱处理前后 HZSM-5 分子篮的 SEM 照片, 由图 2 可见，未经碱处理的 HZSM-5 分子篮表面较为 光滑; 经过碱溶液处理后的 HZSM-5 分子篮, 表面都 产生了一定程度的缺陷。当采用 $\mathrm{CH}_{3} \mathrm{COONa}$ 溶液处理 HZSM-5 分子篮时, 随着 $\mathrm{CH}_{3} \mathrm{COONa}$ 溶液浓度的增加, HZSM-5 分子篎表面凹陷逐渐加深; 当采用 $\mathrm{Na}_{2} \mathrm{CO}_{3}$ 溶 液处理 HZSM-5 分子篮时, 分子篮表面出现很多较密 集的凹陷，表明 HZSM-5 分子篮颗粒被腐蚀较严重; 当采用 $\mathrm{NaOH}$ 溶液处理 HZSM-5 分子篮时, HZSM-5 分子篮颗粒的表面被严重腐蚀, 形成很多较长的缝隙, 但仍保持基本的 HZSM-5 分子篮晶体形貌。与常规碱 溶液相比，采用 $\mathrm{CH}_{3} \mathrm{COONa}$ 溶液处理 HZSM-5 分子篮, 对分子篮形貌结构影响较小，处理深度容易控制，具 有较好的可调控性, 这与 XRD 表征结果相符。
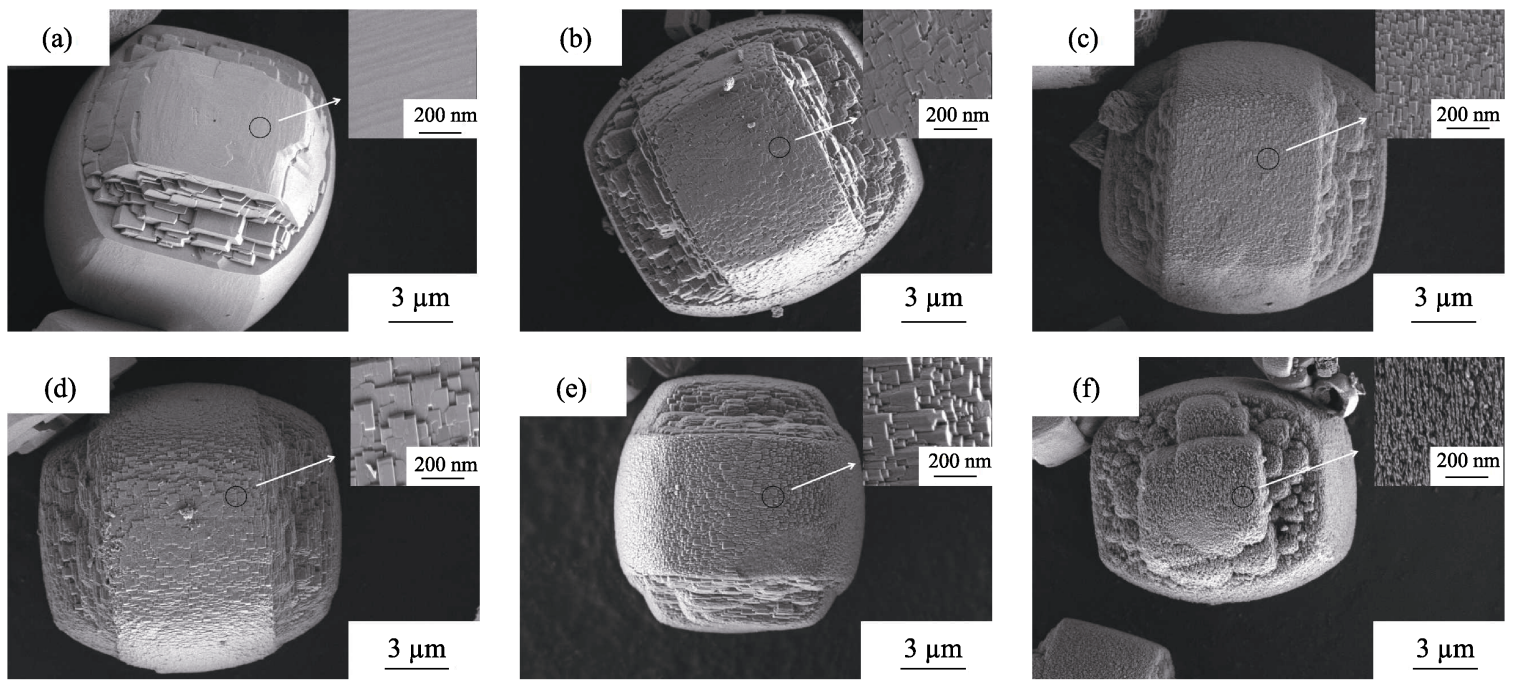

图 2 碱处理前后 HZSM-5 分子篎的 SEM 照片

Fig. 2 SEM images of HZSM-5 zeolites before and after alkaline treatment a: HZSM-5(0); b: HZSM-5(CH $\left.\mathrm{CH}_{3} \mathrm{COONa}, 2.0\right)$; c: HZSM-5( $\left.\mathrm{CH}_{3} \mathrm{COONa}, 4.0\right)$; d: HZSM-5( $\left.\mathrm{CH}_{3} \mathrm{COONa}, 6.0\right)$; e: $\mathrm{HZSM}-5\left(\mathrm{Na}_{2} \mathrm{CO}_{3}, 2.0\right)$; f: HZSM-5(NaOH, 1.0$)$ 


\section{3 碱处理对 HZSM-5 分子篮孔结构的影响}

图 3 为碱处理前后 HZSM-5 分子笁的 $\mathrm{N}_{2}$ 吸附-脱 附等温线, 由图 3 可知, 未使用碱溶液处理时, 样品中 没有出现明显的滞后环, 属于典型的微孔结构等温曲 线; 使用碱溶液处理时, HZSM-5 分子篮在 $p / p_{0}$ 为 0.44 0.97 时呈现出较明显的滞后环, 说明 HZSM-5 分 子篮中引入了介孔结构。其中, 采用 $\mathrm{NaOH}$ 溶液处理 的 HZSM-5 分子篎形成的滞后环最大, $\mathrm{Na}_{2} \mathrm{CO}_{3}$ 溶液处 理 HZSM-5 分子篮形成的滞后环次之, $\mathrm{CH}_{3} \mathrm{COONa}$ 溶 液处理 HZSM-5 分子篮形成的滞后环最小, 且随着 $\mathrm{CH}_{3} \mathrm{COONa}$ 溶液浓度的增加, 形成的滞后环逐渐增大, 说明随着碱性和碱浓度的提高, 生成的介孔数量逐渐 增多, 孔径逐渐增大。

碱溶液处理对 HZSM-5 分子篎的孔结构数据见表 2 。 随着 $\mathrm{CH}_{3} \mathrm{COONa}$ 溶液浓度的增加, 样品的介孔体积和 平均孔径变大, 说明随着碱浓度的增加, 碱溶液体系 对 HZSM-5 分子篮处理深度逐渐加深, 形成的介孔结 构逐渐增多; 总比表面积和外比表面积先增大后减小, 这是因为 $\mathrm{CH}_{3} \mathrm{COONa}$ 溶液能够腐蚀 HZSM-5 分子篎表 面, 分子篎表面逐渐由平滑变得粗粘, 因此样品的外 比表面积和总比表面积逐渐增大, 但当采用 $6.0 \mathrm{~mol} / \mathrm{L}$ 的 $\mathrm{CH}_{3} \mathrm{COONa}$ 溶液处理 HZSM-5 分子笁后, 分子篮表 面凹陷较深, 因此, HZSM-5 $\left(\mathrm{CH}_{3} \mathrm{COONa}, 6.0\right)$ 分子篮的

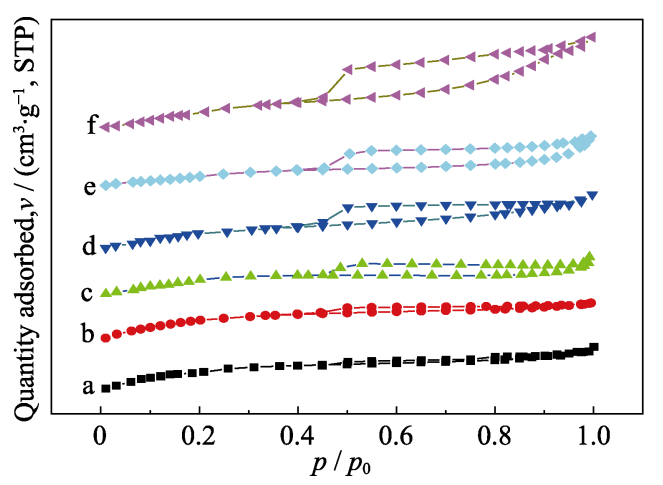

图 3 碱处理前后 HZSM-5 分子篮的 $\mathrm{N}_{2}$ 吸附-脱附等温线 Fig. $3 \mathrm{~N}_{2}$ adsorption-desorption isotherms of HZSM-5 zeolites before and after alkaline treatment a: HZSM-5(0); b: HZSM-5(CH $\left.\mathrm{CH}_{3} \mathrm{COONa}, 2.0\right)$; c: $\mathrm{HZSM}-5\left(\mathrm{CH}_{3} \mathrm{COONa}\right.$, 4.0); d: HZSM-5( $\left.\mathrm{CH}_{3} \mathrm{COONa}, 6.0\right)$; e: $\mathrm{HZSM}-5\left(\mathrm{Na}_{2} \mathrm{CO}_{3}, 2.0\right)$; f: HZSM$5(\mathrm{NaOH}, 1.0)$
外比表面积和总比表面积减小。与常规碱溶液 $\left(\mathrm{Na}_{2} \mathrm{CO}_{3}\right.$ 和 $\mathrm{NaOH}$ 溶液)相比, 采用 $\mathrm{CH}_{3} \mathrm{COONa}$ 溶液处理 HZSM-5 分子篮与未经碱溶液处理的 HZSM-5 分子篮 的微孔体积相差最小, 说明采用 $\mathrm{CH}_{3} \mathrm{COONa}$ 溶液进行 碱处理对 HZSM-5 分子篮骨架结构的影响较小, 这也 与 XRD 和 SEM 表征结果相符。

\section{4 碱处理对 HZSM-5 分子篮表面酸性的影响}

图 4 为碱处理前后 HZSM-5 分子笁的 $\mathrm{NH}_{3}$-TPD 谱图, 表 3 为样品的相对酸量。由图 4 和表 3 可见, 经 过不同碱溶液处理前后的 HZSM-5 分子篮样品都呈现 出两种强度不同的酸性中心, 在 $150 \sim 200{ }^{\circ} \mathrm{C}$ 的脱附峰 对应的是 HZSM-5 分子篮的弱酸中心, 在 $360 \sim 420{ }^{\circ} \mathrm{C}$ 的脱附峰对应的是 HZSM-5 分子篮强酸中心。随着 $\mathrm{CH}_{3} \mathrm{COONa}$ 溶液浓度的增加, HZSM-5 分子篮的酸强 度和相对酸量均呈现出先降低后升高的趋势。当 $\mathrm{CH}_{3} \mathrm{COONa}$ 溶液浓度为 $4.0 \mathrm{~mol} / \mathrm{L}$ 时, HZSM-5 分子篮 的酸强度和相对酸量最低, 这是由于 HZSM-5 分子篮 的酸性主要来源于骨架上和孔隙中的三配位铝原子和 铝离子 $(\mathrm{AlO})^{+}$, 当采用较低浓度的 $\mathrm{CH}_{3} \mathrm{COONa}$ 溶液处 理 HZSM-5 分子篎时, 主要脱除其非骨架硅和铝, 此 时酸强度和酸量的下降与脱除非骨架铝有关 ${ }^{[17]}$; 当采 用 $6.0 \mathrm{~mol} / \mathrm{L} \mathrm{CH}_{3} \mathrm{COONa}$ 溶液处理 HZSM-5 分子篮时, 开始选择性地溶解骨架硅原子, $\mathrm{Si}-\mathrm{O}-\mathrm{Si}$ 键断裂, 初级 单元开始从本体脱落, 该脱硅过程见图 5, 而 HZSM-5 分子筛骨架中的 $\mathrm{Si}-\mathrm{O}-\mathrm{Al}$ 键不易水解断裂, 在这一过 程中分子篮骨架脱铝速率较慢且脱除的数量较少, 因 此 HZSM-5 分子篮骨架中的铝含量相对较多, 使得分 子篮的酸强度和相对酸量升高。

当采用 $\mathrm{Na}_{2} \mathrm{CO}_{3}$ 和 $\mathrm{NaOH}$ 溶液处理 HZSM-5 分子 篮时, 分子篮的酸强度和相对酸量均显著增大, 这是 因为 $\mathrm{Na}_{2} \mathrm{CO}_{3}$ 和 $\mathrm{NaOH}$ 溶液碱性较强, 碱溶液体系直接 溶解分子篎骨架硅原子, 骨架铝含量相对增加, 分子 笁的酸强度和相对酸量升高。

\section{5 碱处理对催化剂表面 Pt 分散度的影响}

表 4 为不同碱溶液处理前后的 Pt/HZSM-5 催化剂 样品的氢吸附量和 Pt 分散度。由表 4 可见, 与未使用碱 溶液处理的 Pt/HZSM-5 催化剂样品相比, 采用碱溶液处 理后, $\mathrm{Pt}$ 分散度都有一定程度的增加。当采用 $\mathrm{CH}_{3} \mathrm{COONa}$

表 2 碱溶液处理对 HZSM-5 分子篮结构性质的影响

Table 2 Effect of alkaline treatment on structure of HZSM-5 zeolites

\begin{tabular}{|c|c|c|c|c|c|}
\hline Sample & $A_{\mathrm{BET}} /\left(\mathrm{m}^{2} \cdot \mathrm{g}^{-1}\right)$ & $A_{\text {ext }} /\left(\mathrm{m}^{2} \cdot \mathrm{g}^{-1}\right)$ & $v_{\text {micro }} /\left(\mathrm{cm}^{3} \cdot \mathrm{g}^{-1}\right)$ & $v_{\text {meso }} /\left(\mathrm{cm}^{3} \cdot \mathrm{g}^{-1}\right)$ & $d_{\text {aver }} / \mathrm{nm}$ \\
\hline HZSM-5(0) & 357.92 & 29.98 & 0.17 & 0.04 & 1.52 \\
\hline $\mathrm{HZSM}-5\left(\mathrm{CH}_{3} \mathrm{COONa}, 2.0\right)$ & 366.64 & 74.43 & 0.16 & 0.07 & 2.35 \\
\hline $\mathrm{HZSM}-5\left(\mathrm{CH}_{3} \mathrm{COONa}, 4.0\right)$ & 378.69 & 103.83 & 0.16 & 0.11 & 2.93 \\
\hline HZSM-5 $\left(\mathrm{CH}_{3} \mathrm{COONa}, 6.0\right)$ & 375.72 & 93.98 & 0.15 & 0.14 & 3.26 \\
\hline HZSM-5 $\left(\mathrm{Na}_{2} \mathrm{CO}_{3}, 2.0\right)$ & 372.64 & 85.48 & 0.13 & 0.17 & 3.51 \\
\hline HZSM-5(NaOH, 1.0) & 360.23 & 66.25 & 0.08 & 0.28 & 4.67 \\
\hline
\end{tabular}




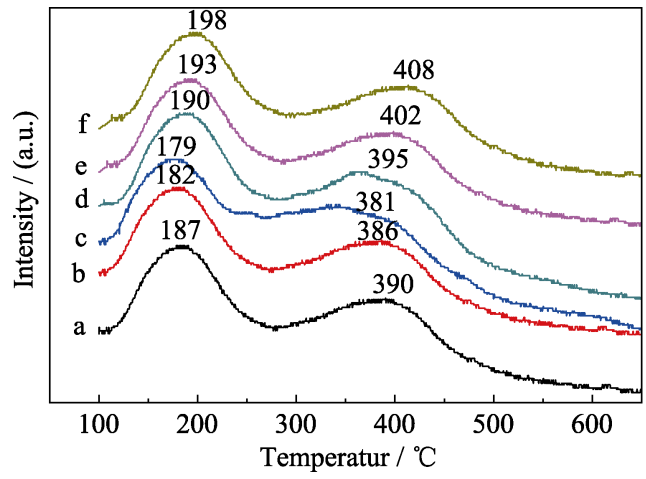

图 4 碱处理前后 HZSM-5 分子篮的 $\mathrm{NH}_{3}-\mathrm{TPD}$ 曲线

Fig. $4 \mathrm{NH}_{3}$-TPD curves of HZSM-5 zeolites before and after alkaline treatment

a: HZSM-5(0); b: HZSM-5( $\left.\mathrm{CH}_{3} \mathrm{COONa}, 2.0\right)$; c: HZSM-5( $\mathrm{CH}_{3} \mathrm{COONa}$, 4.0); d: $\mathrm{HZSM}-5\left(\mathrm{CH}_{3} \mathrm{COONa}, 6.0\right)$; e: $\operatorname{HZSM}-5\left(\mathrm{Na}_{2} \mathrm{CO}_{3}, 2.0\right)$; f: HZSM-5 $(\mathrm{NaOH}, 1.0)$

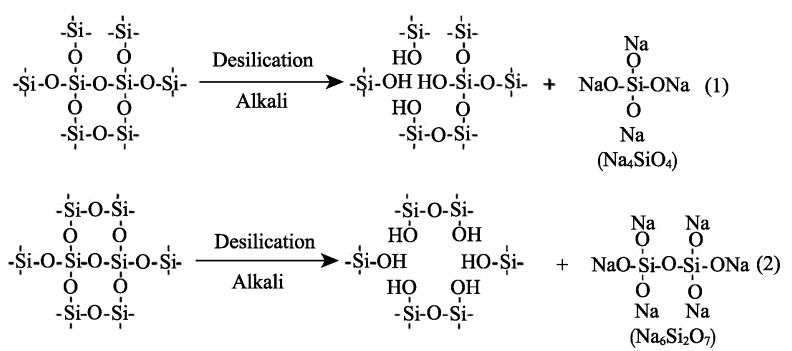

图 5 碱处理 HZSM-5 分子篮的脱硅过程

Fig. 5 Desilication process of the parent HZSM-5 zeolite during the alkali treatment

溶液处理 HZSM-5 分子篮时, 随着 $\mathrm{CH}_{3} \mathrm{COONa}$ 溶液浓 度的增加, $\mathrm{Pt}$ 分散度先增大后减小, 当 $\mathrm{CH}_{3} \mathrm{COONa}$ 溶 液浓度为 $4.0 \mathrm{~mol} / \mathrm{L}$ 时, $\mathrm{Pt}$ 分散度最高可达 $52.9 \%$ 。由 SEM 分析可知, HZSM-5 分子篎经过碱溶液处理后, 表面出现一定程度的缺陷, 且随着 $\mathrm{CH}_{3} \mathrm{COONa}$ 溶液浓 度的增加, 分子篮的表面粗䊁程度逐渐增大, 因此, 采用 $2.0 \sim 4.0 \mathrm{~mol} / \mathrm{L}$ 的 $\mathrm{CH}_{3} \mathrm{COONa}$ 溶液处理 $\mathrm{HZSM}-5$ 分子篮, 样品表面的可利用区域增加, 活性组分 $\mathrm{Pt}$ 在 分子篮表面的分散趋于均匀; 而采用 $6.0 \mathrm{~mol} / \mathrm{L}$ 的 $\mathrm{CH}_{3} \mathrm{COONa}$ 溶液和常规碱溶液 $\left(\mathrm{Na}_{2} \mathrm{CO}_{3}\right.$ 和 $\mathrm{NaOH}$ 溶液 $)$ 处理分子篎, 样品表面粗糛程度进一步增加, 分子篎 表面形成较深的凹陷, 导致活性组分 Pt 趋向聚集。因 此, Pt 分散度下降。

\section{6 催化剂的丙烷脱氢性能}

图 6 与图 7 分别为不同碱溶液处理前后的
Pt/HZSM-5 催化剂样品的丙烷转化率和丙烯选择性与 反应时间的关系曲线。由图 6 和图 7 可见, 不同碱溶 液处理前后的 Pt/HZSM-5 催化剂样品的丙烷初始转化 率均高于 $30 \%$, 丙烯选择性均远高于 $84 \%$, 说明采用 HZSM-5 分子篮作为丙烷脱氢催化剂载体, 其反应性 能优于工业上以 $\mathrm{Al}_{2} \mathrm{O}_{3}$ 作为催化剂载体的反应性能。 由图 6 可见, 经过不同碱溶液处理后的 Pt/HZSM-5 催 化剂样品的丙烷初始转化率都较高, 但随着反应时间 的延长, 丙烷转化率有一定程度的下降, 其中, 随着 $\mathrm{CH}_{3} \mathrm{COONa}$ 溶液浓度的增加, 丙烷初始转化率呈现出 先增大后减小的趋势。由图 7 可见，随着反应时间的 延长, 样品的丙烯选择性均增大, 且随着 $\mathrm{CH}_{3} \mathrm{COONa}$ 溶液浓度的增加, 丙烯选择性先增大后减小。值得指出 的是, 当采用 $4.0 \mathrm{~mol} / \mathrm{L}$ 的 $\mathrm{CH}_{3} \mathrm{COONa}$ 溶液处理时, 催 化剂样品的丙烷初始转化率和丙烯选择性均达到最佳 状态。

Pt/HZSM-5 催化剂属于双功能催化剂, 即活性组 分 Pt 的金属功能和 HZSM-5 分子篮的酸功能，两者之 间存在协同效应，共同影响丙烷脱氢催化剂的反应性 能 ${ }^{[15]}$ 。其中, 负载型 $\mathrm{Pt}$ 催化剂表面存在两类活性中心 M1 和 M2, M1 中心适合烃类裂解和氢解反应; M2 中心 适合烷烃脱氢反应。丙烷脱氢反应机理如图 8 所示, $\mathrm{C}_{3} \mathrm{H}_{8}$ 首先吸附在 HZSM-5 载体上, 然后在 HZSM-5 分 子篮表面的酸性位点上生成 $\mathrm{C}_{3} \mathrm{H}_{7}$ 自由基和 $\mathrm{H}$ 自由基, 最后在活性中心 M2 的作用下生成 $\mathrm{H}_{2}$ 和目的产物 $\mathrm{C}_{3} \mathrm{H}_{6}$ 。为了使 HZSM-5 分子篎的酸功能和 $\mathrm{Pt}$ 的金属功 能更好地发展协同作用, 要求两种活性中心靠得很近, 既需要 Pt 在 HZSM-5 分子篮上处于高度分散的状态。

由图 3 可知, 常规 HZSM-5 分子篮孔径属于微孔 范围, 微孔孔径会限制反应物和产物的进出, 增大了 传质阻力, 并且由于 $\mathrm{Pt}$ 的分散度相对较低, 所以未经 碱溶液处理的 Pt/HZSM-5(0)催化剂样品的丙烷初始转 化率较低且下降较快; 而碱溶液处理, 一方面, 扩大 了 HZSM-5 分子篮的外比表面积, 表面出现缺陷, 这 在一定程度上提高了 $\mathrm{Pt}$ 的分散度; 另一方面, 在碱溶 液的作用下，产生了部分介孔，改善了扩散限制，缩 短了烃类物质在催化剂上的停留时间, 减少了表面积 碳，因而提高了丙烷脱氢反应活性。由 HZSM-5 分子 篮的结构性质和 $\mathrm{Pt}$ 的分散度可见, 采用 $4.0 \mathrm{~mol} / \mathrm{L}$ 的 $\mathrm{CH}_{3} \mathrm{COONa}$ 溶液处理 HZSM-5 分子篎, 不仅形成了微

表 3 碱处理前后 HZSM-5 分子篮的相对酸量

Table 3 Relative acid amount of HZSM-5 zeolites before and after alkaline treatment

\begin{tabular}{|c|c|c|c|c|c|c|}
\hline \multirow{2}{*}{$\begin{array}{c}\text { Acid } \\
\text { strength }\end{array}$} & \multicolumn{6}{|c|}{ Relative acid amount $/ \%$} \\
\hline & HZSM-5(0) & $\begin{array}{c}\text { HZSM-5 } \\
\left(\mathrm{CH}_{3} \mathrm{COONa}, 2.0\right)\end{array}$ & $\begin{array}{c}\text { HZSM-5 } \\
\left(\mathrm{CH}_{3} \mathrm{COONa}, 4.0\right)\end{array}$ & $\begin{array}{c}\text { HZSM-5 } \\
\left(\mathrm{CH}_{3} \mathrm{COONa}, 6.0\right)\end{array}$ & $\begin{array}{c}\text { HZSM-5 } \\
\left(\mathrm{Na}_{2} \mathrm{CO}_{3}, 2.0\right)\end{array}$ & $\begin{array}{c}\text { HZSM-5 } \\
(\mathrm{NaOH}, 1.0)\end{array}$ \\
\hline Strong & 100 & 98.17 & 90.83 & 102.25 & 103.47 & 108.15 \\
\hline Weak & 100 & 98.14 & 90.06 & 101.86 & 103.18 & 107.28 \\
\hline
\end{tabular}


表 4 碱溶液处理前后催化剂的氢吸附量和 Pt 分散度分析 Table 4 Analysis of hydrogen adsorption amount and Pt dispersion of catalysts before and after alkaline treatment

\begin{tabular}{ccc}
\hline Catalyst & $\begin{array}{c}\text { Hydrogen } \\
\text { chemisorption/ } \\
\left(\mathrm{mL} \cdot \mathrm{g}^{-1} \mathrm{Pt}\right)\end{array}$ & $\begin{array}{c}\text { Bare } \\
\text { fraction } \\
\text { of metallic } \\
\mathrm{Pt} / \%\end{array}$ \\
\hline $\mathrm{Pt} / \mathrm{HZSM}-5(0)$ & 15.0 & 34.8 \\
$\mathrm{Pt} / \mathrm{HZSM}-5\left(\mathrm{CH}_{3} \mathrm{COONa}, 2.0\right)$ & 21.7 & 50.4 \\
$\mathrm{Pt} / \mathrm{HZSM}-5\left(\mathrm{CH}_{3} \mathrm{COONa}, 4.0\right)$ & 22.8 & 52.9 \\
$\mathrm{Pt} / \mathrm{HZSM}-5\left(\mathrm{CH}_{3} \mathrm{COONa}, 6.0\right)$ & 22.2 & 51.6 \\
$\mathrm{Pt} / \mathrm{HZSM}-5\left(\mathrm{Na}_{2} \mathrm{CO}_{3}, 2.0\right)$ & 19.5 & 45.2 \\
$\mathrm{Pt} / \mathrm{HZSM}-5\left(\mathrm{NaOH}_{1}, 1.0\right)$ & 16.6 & 38.6 \\
\hline
\end{tabular}

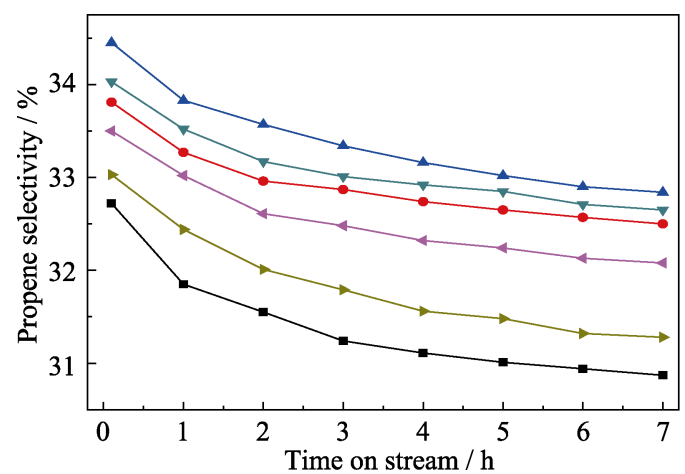

图 6 不同催化剂的丙烷转化率随反应时间的变化关系

Fig. 6 Relationship between propane conversion and reaction time for different catalysts

a: $\mathrm{Pt} / \mathrm{HZSM}-5(0)$; •: Pt/HZSM-5( $\left.\mathrm{CH}_{3} \mathrm{COONa}, 2.0\right) ; \boldsymbol{\Delta}: \mathrm{Pt} / \mathrm{HZSM}-5$ $\left(\mathrm{CH}_{3} \mathrm{COONa}, 4.0\right) ; \boldsymbol{\nabla}: \mathrm{Pt} / \mathrm{HZSM}-5\left(\mathrm{CH}_{3} \mathrm{COONa}, 6.0\right)$ ४ $\mathrm{Pt} / \mathrm{HZSM}-5$ $\left(\mathrm{Na}_{2} \mathrm{CO}_{3}, 2.0\right)$; : Pt/HZSM-5(NaOH, 1.0)

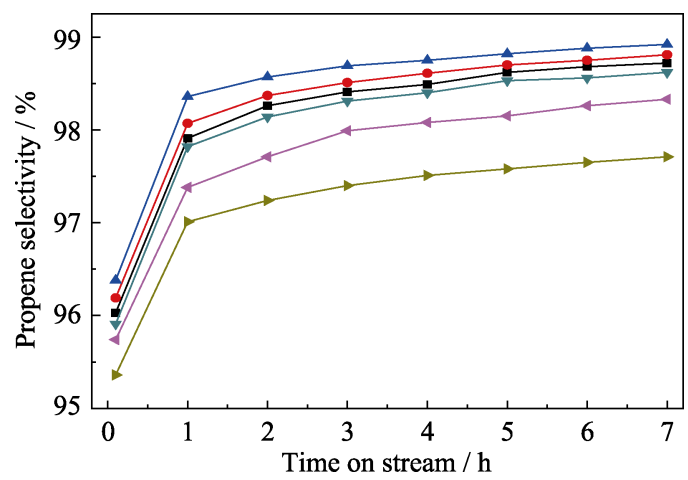

图 7 不同催化剂的丙烯选择性随反应时间的变化关系

Fig. 7 Relationship between propene selectivity and reaction time for different catalysts

a: $\mathrm{Pt} / \mathrm{HZSM}-5(0)$; •: Pt/HZSM-5(CH$\left.{ }_{3} \mathrm{COONa}, 2.0\right) ; \boldsymbol{\Delta}: \mathrm{Pt} / \mathrm{HZSM}-5$ $\left(\mathrm{CH}_{3} \mathrm{COONa}, 4.0\right) ; \boldsymbol{\nabla}: \mathrm{Pt} / \mathrm{HZSM}-5\left(\mathrm{CH}_{3} \mathrm{COONa}, 6.0\right)$ ४ $\mathrm{Pt} / \mathrm{HZSM}-5$ $\left(\mathrm{Na}_{2} \mathrm{CO}_{3}, 2.0\right)$; : Pt/HZSM-5(NaOH, 1.0)

孔-介孔多级孔结构, 而且活性组分 Pt 在分子篮上的 分散度最高，因此，丙烷初始转化率最高，可达 $34.5 \%$; 而采用常规碱溶液 $\left(\mathrm{Na}_{2} \mathrm{CO}_{3}\right.$ 和 $\mathrm{NaOH}$ 溶液) 处理 HZSM-5 分子篮, 虽然能够形成微孔-介孔多级孔结构, 但对分子篮的孔结构影响较大, 且分子篮表面腐蚀程 度较深，导致 Pt 分散度下降，不利于反应活性的提高， 因此丙烷初始转化率又转向降低。

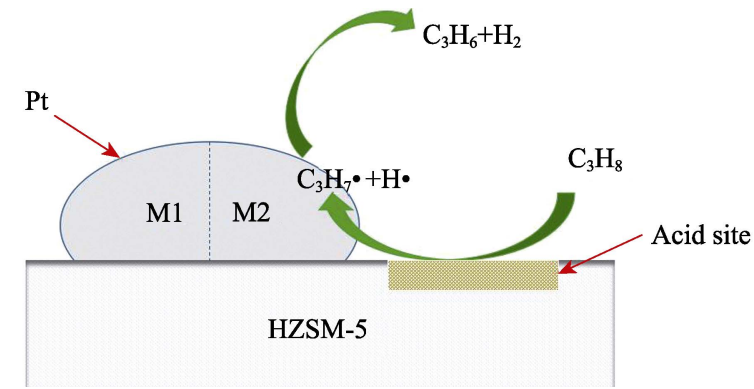

图 8 丙烷脱氢反应机理示意图

Fig. 8 Schematic diagram of mechanism of dehydrogenation of propane

丙烷分子在 Pt/HZSM-5 催化剂表面通过两种途径 形成积碳，一是在活性组分 Pt 表面上的氢解和裂解过 程; 二是在 HZSM-5 载体酸中心上的聚合和环化过 程。已有的研究结果表明, 积碳会优先覆盖 Pt/HZSM-5 的 M1 活性中心，造成 M1 中心减少，而 M1 活性中心 参与氢解和裂解反应, 因此氢解和裂解反应会受到抑 制，丙烯选择性会随反应的进行而逐渐增强。另外，采 用适当浓度的 $\mathrm{CH}_{3} \mathrm{COONa}$ 溶液处理 HZSM-5 分子篎，能 够有效降低分子篮的表面酸性，使积碳前驱体在催化 剂酸性表面转化和在催化剂表面上的沉积受到抑制, 从而使丙烯选择性进一步增强，因此，Pt/HZSM-5 $\left(\mathrm{CH}_{3} \mathrm{COONa}, 4.0\right)$ 催化剂样品的丙烯选择性最高达 $98.9 \%$ 。而采用常规碱溶液 $\left(\mathrm{Na}_{2} \mathrm{CO}_{3}\right.$ 和 $\mathrm{NaOH}$ 溶液)处 理时，分子篮的表面酸性会增强，加速了副反应在 HZSM-5 分子篮表面酸性中心上生成积碳，使得丙烯 选择性在一定程度上有所下降。

\section{3 结论}

1) 与常规碱溶液 $\left(\mathrm{Na}_{2} \mathrm{CO}_{3}\right.$ 和 $\mathrm{NaOH}$ 溶液)相比, 采 用 $\mathrm{CH}_{3} \mathrm{COONa}$ 溶液处理 HZSM-5 分子篮，在对分子筛 的晶体结构和表面形貌影响较小的基础上, 有效地引 入了介孔结构，调节了 HZSM-5 分子篮的酸性，通过 适度增加表面缺陷，提高了 $\mathrm{Pt}$ 分散度。

2) 随着 $\mathrm{CH}_{3} \mathrm{COONa}$ 溶液浓度的增加，样品的丙 烷转化率和丙烯选择性均呈现出先增大后减小的趋势, 与常规碱溶液 $\left(\mathrm{Na}_{2} \mathrm{CO}_{3}\right.$ 和 $\mathrm{NaOH}$ 溶液)相比, 采用 $\mathrm{CH}_{3} \mathrm{COONa}$ 溶液处理制备的 Pt/HZSM-5 催化剂具有更 高的丙烷脱氢反应活性和选择性。当 $\mathrm{CH}_{3} \mathrm{COONa}$ 溶液 浓度为 $4.0 \mathrm{~mol} / \mathrm{L}$ 时, 丙烷初始转化率和丙烯选择性均 达到最高值，分别为 $34.5 \%$ 和 $98.9 \%$ 。

\section{参考文献:}

[1] ZHAO DAN, YUAN MEI-HUA, ZHANG YAO-YUAN, et al. Incorporation of $\mathrm{Cr}$ in ZSM-5 zeolite framework as bifunctional catalysts for $n$-butane catalytic cracking. CIESC Journal, 2016, 67(8): 3400-3407. 
[2] ZHANG YI-WEI, ZHOU YU-MING, SHI JUN-JUN, et al. Comparative study of bimetallic Pt-Sn catalysts supported on different supports for propane dehydrogenation. Journal of Molecular Catalysis A: Chemical, 2014, 381: 138-147.

[3] KUMAR M SANTHOSH, CHEN DE, HOLMEN ANDERS, et al. Dehydrogenation of propane over Pt-SBA-15 and Pt-Sn-SBA-15: effect of $\mathrm{Sn}$ on the dispersion of Pt and catalytic behavior. Catalysis Today, 2009, 142(1/2): 17-23.

[4] QIU AN-DING, LI EN-XIA, FAN YI-NING. Effect on catalytic performance of PtSn/ZSM-5 catalyst for propane dehydrogenation. Chinese Journal of Catalysis, 2007, 28(11): 970-974.

[5] LI JIAN-HUA, YANG DONG-HUA, LÜ AI-NING, et al. Synthesis and characterization of ZSM-5/EU-1 composite zeolite with core-shell structure by one step. Journal of Inorganic Materials, 2016, 31(5): 492-498.

[6] ZHANG YI-WEI, ZHOU YU-MING, TANG MENG-HAN, et al. Effect of La calcination temperature on catalytic performance of PtSnNaLa/ZSM-5 catalyst for propane dehydrogenation. Chemical Engineering Journal, 2012, 181-182: 530-537.

[7] SHEINTUCH M, LIRON O, RICCA A, et al. Propane dehydrogenation kinetics on supported Pt catalyst. Applied Catalysis A: General, 2016, 516: 17-29.

[8] MASARU OGURA, SHIN-YA SHINOMIYA, JUNKO TATENO, et al. Alkali-treatment technique-new method for modification of structural and acid-catalytic properties of ZSM-5 zeolites. Applied Catalysis A:General, 2001, 219: 33-43.

[9] FATHI S, SOHRABI M, FALAMAKI C. Improvement of HZSM-5 performance by alkaline treatments: comparative catalytic study in the MTG reactions. Fuel, 2014, 116: 529-537.

[10] SHI GANG, LIN XIU-YING, FAN YU, et al. Desilication modifi- cation of ZSM-5 zeolite and its catalytic properties in hydro-upgrading. Journal of Fuel Chemistry and Technology, 2013, 41(5): 589-560.

[11] LIU DONG-MEI, ZHAI YU-CHUN, MA JIAN, et al. Preparation of micro-mesoporous ZSM-5 modified by $\mathrm{Na}_{2} \mathrm{CO}_{3}$ and its catalytic performance for sulfur etherification. Acta Petrolei Sinica (Petroleum Processing Section), 2015, 31(1): 38-44.

[12] GAO XIONG-HOU, TANG ZHI-CHENG, LU GONG-XUAN, et al. Butene catalytic cracking to ethylene and propylene on mesoporous ZSM-5 by desilication. Solid State Sciences, 2010, 12: 1278-1282.

[13] OGURA M, SHINOMIYA S. Alkali-treatment technique-new method for modification of structural and acid-catalytic properties of ZSM-5 zeolites. Applied Catalysis A: General, 2001, 219(3): 33-43.

[14] HAO CHEN, HAO CHENG, FANG ZHOU, et al. Catalytic fast pyrolysis of rice straw to aromatic compounds over hierarchical HZSM-5 produced by alkali treatment and metal-modification. Journal of Analytical and Applied Pyrolysis, 2018, 131: 76-84.

[15] ZHANG YI-WEI, ZHOU YU-MING, QIU AN-DING, et al. Effect of $\mathrm{Na}$ addition on catalytic performance of $\mathrm{PtSn} / \mathrm{ZSM}-5$ catalyst for propane dehydrogenation. Acta Phys.-Chim. Sin., 2006, 22(6): $672-678$.

[16] SUZUKI TETSUO, OKUHARA TOSHIO. Change in pore structure of MFI zeolite by treatment with $\mathrm{NaOH}$ aqueous solution. $\mathrm{Mi}$ croporous and Mesoporous Materials, 2001, 43(1): 83-89.

[17] SONG YUE-QIN, FENG YAN-LONG, LIU FENG, et al. Effect of variations in pore structure and acidity of alkali treated ZSM-5 on the isomerization performance. Journal of Molecular Catalysis A: Chemical, 2009, 310(1/2): 130-137. 